\title{
Neutrophil to Lymphocyte Ratio as Predictive Marker of Recurrence of HCC after Radiofrequency Ablation
}

\author{
Eman M. Abdel Rahman', Fatma M. EL-Senosy ${ }^{1, *}$, Ayman Alsebaey ${ }^{2}$, Shiamaa A. Hussien ${ }^{1}$ \\ ${ }^{1}$ Department of internal medicine, faculty of Medicine (Girls), Al Azher University, Cairo, Egypt \\ ${ }^{2}$ Department of Hepatology, National Liver Institute, Menoufia University, Shebeen El-Kom, Egypt \\ *Corresponding author: fat_sen@yahoo.com
}

Received November 17, 2018; Revised December 28, 2018; Accepted January 10, 2019

\begin{abstract}
Background: Neutrophil to lymphocyte ratio (NLR), platelet to lymphocyte ratio (PLR), and prognostic nutritional index (PNI), are immunonutritional indices, have been shown as an independent factor to predict postoperative recurrence and/or overall survival in patients with hepatocellular carcinoma (HCC). Aim: to validate neutrophils-to-lymphocytes ratio (NLR) as a predictor of post radiofrequency ablation recurrence of hepatocellular carcinoma (HCC). Patients and methods: This cross sectional prospective study was conducted on 50 Egyptian patients had radiofrequency ablation for HCC within Barcelona clinical liver cancer (BCLC) stage A. Pre-treatment laboratory tests and imaging were used to measure NLR, PLR, PNI, Child-Pugh (CTP score, tumor number and tumor size. HCC recurrences were followed after three month. Results: According to NLR cut-off value, the patients with NLR $>1.73$ had larger tumour size, and higher rates of tumor multiplicity .After 3 month follow up $30 / 50(60 \%)$ patients had tumour recurrence. Regarding recurrence after RFA showed that CTP class $\mathrm{B}(\mathrm{P}<0.05)$, tumor size $(\mathrm{P}<0.05)$, higher rates of tumor multiplicity $(\mathrm{P}<0.05)$, decrease PNI $(\mathrm{P}<0.001)$ and increased NLR $(\mathrm{P}<0.05)$ after RFA and at baseline were found to be worse prognosis. A receiver-operating characteristic (ROC) analysis was used to classify patients as follows: NLR-PNI 0 group (NLR $\leq 1.73$ and PNI $>11.74$ ), NLR-PNI 1 group $(\mathrm{NLR}>1.73$ or PNI $\leq 11.74)$ and NLR-PNI 2 group (NLR $>1.73$ and PNI $\leq 11.74)$. The patients with NLR-PNI 2 group had increase in number of portahepatis lymph node, number and size of tumor, decrease serum albumin and change in PNLR. Multivariate analyses suggested increased NLR (hazard ratio [HR] =2.09; 95\% confidence interval $[\mathrm{CI}]=1.88-2.55 ; \mathrm{P}<0.05)$, increased PLR $(\mathrm{HR}=0.07 ; 95 \% \mathrm{CI}=0.06-0.08 ; \mathrm{P}<0.05)$, and increased AFP $(\mathrm{HR}=59.20$; 95\% CI=-99.74-638.78; $\mathrm{P}<0.05$ ) contributed to post-RFA mortality. Conclusion: High blood NLR after RFA is a predictor for worse survival and also can predict recurrence of HCC. Higher NLR-PNI score predict a worse prognosis in patients who underwent RFA.
\end{abstract}

Keywords: hepatocellular carcinoma, Radio Frequency Ablation (RFA), neutrophil to lymphocyte ratio

Cite This Article: Eman M. Abdel Rahman, Fatma M. EL-Senosy, Ayman Alsebaey, and Shiamaa A. Hussien, "Neutrophil to Lymphocyte Ratio as Predictive Marker of Recurrence of HCC after Radiofrequency Ablation.” American Journal of Clinical Medicine Research, vol. 7, no. 1 (2019): 1-8. doi: 10.12691/ajcmr-7-1-1.

\section{Introduction}

Hepatocellular carcinoma (HCC) represents about 90\% of primary liver cancers and is the sixth most frequent malignancy and the third most common cancer-related cause of death worldwide [1]. Despite advanced improvements in therapeutic modalities, the long-term outcomes remain unsatisfactory due to high incidence of tumor recurrence [2].

In the treatment of hepatocellular carcinoma (HCC), fewer than $40 \%$ of patients are candidates for surgery, and the rate of recurrence after curative surgery is high [3].

Percutaneous Radio Frequency Ablation (RFA) is one of the most widely used local ablation therapies for HCC, with a high complete ablation rate of $>85 \%$ for solitary tumors $<5 \mathrm{~cm}$ in diameter or up to 3 tumors with a maximum diameter of $3 \mathrm{~cm}[4,5]$. Also it is an exciting approach to destroying inoperable primary tumors or metastases in the liver [3].

Two previous randomized controlled trials have suggested that RFA is as effective as resection in terms of overall survival (OS) and disease free survival times [6,7].

However, RFA is associated with a high incidence of postoperative recurrence. It has been reported that the cumulative 5-year recurrence rate of patients undergoing RFA is $>70 \%[8,9]$.

Systemic inflammatory response was considered as an important factor influencing HCC recurrence and/or overall survival for patients with HCC following liver resection, liver transplantation, or RFA [8].

Some inflammation-based prognostic systems, such as neutrophil to lymphocyte ratio (NLR), platelet to lymphocyte ratio (PLR), prognostic nutritional index (PNI), and so forth, have been developed by previous investigations to predictive postoperative recurrence and/or overall survival [9]. 
An elevated ratio of peripheral neutrophils-to-lymphocytes (NLR) has been recognized as a poor prognostic indicator in various cancers. However, the consistency and magnitude of the prognostic impact of NLR are unclear [10].

Aim of the work: To validate neutrophils-to-lymphocytes ratio (NLR) as a predictor of post radiofrequency ablation (RFA) recurrence of HCC.

\section{Patients and Methods}

This perspective study was conducted on 50 Egyptian patients with HCC. They were selected from patients attending the HCC clinic and received RFA as initial treatment in Department of Radiology at National Liver Institute Menoufia University during the period from September 2016 and November 2017. This study was conducted at Faculty of medicine "Gilrs", AL-Azhar University and HCC multidisciplinary clinic, hepatology department, National Liver Institute, Menoufia University. An informed consent was obtained from all patients before getting them involved in the study. Also approval of ethical committee of faculty of medicine Al-Azhar University was obtained.

The diagnosis of HCC was carried out according to the American Association for the Study of Liver Diseases (AASLD) Practice Guidelines that proposed that one imaging technique (CT or MRI) showing the HCC radiological hallmark suffices for diagnosing tumors above $1 \mathrm{~cm}$ in diameter in cirrhotic patients [11]. And these patients had a follow-up period of more than 3 months (if patients remained alive).All patients who met the inclusion criteria were enrolled in this study. The Inclusion criteria were: patients with HCC (single or multinodular focal lesion(s), Adequate baseline liver function (Child-Pugh grade A or B), and no clinical symptoms or signs of sepsis.

Patients who fulfilled the following criteria were excluded:Patients with DM, corticostiroid therpy,Obese with body mass index more than $30 \mathrm{Kg} / \mathrm{m} 2$, patients on Immunomedulators treatement and autoimmune hepatitis .

For all patients, demographic information, etiology of liver disease, performance status, the Child-Turcotte-Pugh (CTP) score and its constitutive variables, were recorded. All baseline data were determined at the time of HCC diagnosis and before therapy was instituted.

All patients underwent a thorough assessment of history, complete clinical examination, BMI body weight (kg)/height2(m), liver function tests (Serum albumin, bilirubin, alanine transaminase (ALT) and aspartate transaminase (AST) with calculation of prognostic nutritional index (PNI) international normalized ratio (INR) and Serum Alfa fetoprotein level (AFP), and serum creatinine.

Complete blood picture (CBC) was done on sysmex KN21 with calculation of neutrophil-to-lymphocyte ratio, platelet to lymphocyte ratio and platelet multiple neutrophil to lymphocyte ratio. All of them were done on cobas 311 clinical chemistry auto-analyzer from ROCH diagnostic company.

Imaging examination was done including: Abdominal ultrasonography, Triphasic CT abdomen and or dynamic
MRI abdomen, and Focal lesion biopsy only if the diagnosis is not clear with 2 imaging modalities.

Tumour staging information (including number of focal lesions and maximum diameter of contrast-enhancing lesions), Child-Turcotte-Pugh class [12] and Barcelona Clinic Liver Cancer (BCLC) stage [13], were determined at the beginning of the study for all patients.

Patient with BCLC stage A; single focal lesion $\leq 5 \mathrm{~cm}$ or 3 focal lesions the largest $\leq 3 \mathrm{~cm}$ will undergo radiofrequency ablation.

RFA was performed by using an RFA system (RITA Medical Systems Inc., Mountain View, CA, USA). The access routes were determined by contrast-enhanced computed tomography (CT) or ultrasound. All procedures were performed percutaneously, under general or local anesthetic, by two qualified interventional radiologists with the guidance of real-time ultrasonography or X-ray.

\subsection{Assessment of Therapeutic Responseand Recurrence}

One month after each RFA procedures, imaging examinations including liver US and triphasic CT were performed routinely.A response to RFA was classified as complete ablationindicated by the absence of contrast enhancement in the ablationzone. The new recurrence was assessed after 3 months by US and triphasic CT examination.

Inflammation based index and score. The NLR was defined as the absolute neutrophil count divided by the lymphocyte count [14]. The PNI was calculated as follows: albumin $(\mathrm{g} / \mathrm{L})+0.005 \times$ absolute lymphocyte count $\left(\mathrm{mm}^{3}\right)$ [15].

A receiver-operating characteristic (ROC) analysis was used to classify patients as follows: NLR-PNI 0 group $(\mathrm{NLR} \leq 1.73$ and PNI $>11.47)$, NLR-PNI1 group $(\mathrm{NLR}>1.73$ or $\mathrm{PNI} \leq 11.47)$ and NLR-PNI 2 group $(\mathrm{NLR}>1.73$ and $\mathrm{PNI} \leq 11.47)$.

The PLR was estimated as the absolute platelet count divided by the lymphocyte count. PNLR was calculated as follows: platelet multiply neutrophil divided by lymphocyte count.

Post RFA NLR, PLR, PNI, and PNLR were calculated on the first and three months after RFA. Change of PNLR was calculated by post RFA PNLR minus preRFA PNLR. If the postoperative PNLR minus preoperative PNLR was $>0$, the change of PNLR was considered as increased, if not, it was considered as decreased.

Overall Survival (OS) was assessed. The OS time was defined as the interval between the RFA treatment and the date of death or the last follow up.

\subsection{Statistical Analysis}

Data were collected, revised, coded and entered to the Statistical Package for Social Science (IBM SPSS) version 20. Qualitative data were presented as number and percentages while quantitative data were presented as mean, standard deviations and ranges when parametric. The comparison between two groups with qualitative data were done by using Chi-square test and/or Fisher exact test was used instead of Chi-square test when the expected count in any cell was found less than 5.The comparison 
between two groups regarding quantitative data with parametric distribution was done by using Independent t-test. Spearman correlation coefficients were used to assess the correlation between two quantitative parameters in the same group. The confidence interval was set to $95 \%$ and the margin of error accepted was set to $5 \%$. So, the p-value was considered significant as the following: $\mathrm{P}>0.05$ : Non-significant. $\mathrm{P}<0.05$ : Significant $. \mathrm{P}<0.001$ : Highly significant.

ROC-curve: Receiver Operating Characteristic curve analysis

Sensitivity: Probability that the test results will be positive when the disease is present (true positive rate, expressed as a percentage).

Specificity: Probability that the test results will be negative when the disease is present (true negative rate, expressed as a percentage).

PPV: Positive Predictive value (probability that the disease is present when the test is positive).

NPV: Negative Predictive value (probability that the disease is present when the test is negative).

Accuracy: the ratio of the true positive and true negative on all patients.

\section{Results}

Baseline characteristics: This study was conducted on 50 Egyptian patients with HCC who had undergone RFA as initial treatment. They were 41 males (82\%) and 9 females (18\%) and their ages ranged between (40-70) years with mean (59.52 \pm 7.28 years), and 25 (50\%) patients had a single nodule while 25 (50\%) had multiple nodules. With respect to etiology of HCC there were thirty four patients (68\%) was hepatitis $C$ virus, None of the studied patients had a history of hepatitis $\mathrm{B}$ and in 10 patients (20\%) the etiology was unknown, 32 patients (64\%) gave history of bilharziasis, 29 patients(58\%) received tartar emetic injection. The diameters of the initial tumors ranged from 2.5 to $8.6 \mathrm{~cm}$, with a median of $4 \mathrm{~cm}$. With regard to staging all patients belonged to BCLC stage A. According to Child-Turcotte-Pugh score there was 44 patients (88\%) with Child- "A" while 6 patients $(12 \%)$ Child "B". The diagnosis of HCC was carried out according to the American Association for the Study of Liver Diseases (AASLD) Practice Guidelines that proposed that one imaging technique (CT or MRI).The mean values and SD of the pre RFANLR, PNI, PLR and PNL Rand the median values and ranges of AFP shown in Table 1.

After a follow-up period of 3 months, 30 (60\%) patients had new recurrent tumour (s) where as 10 (20\%) patients died. Among patients with post RFA recurrence we assess the risk factors associated with recurrence as shown in Table 2, our result suggested that increase in number of Portahepatis LN, size and number of Tumour, increase in NLR and change of PNLR after RFA, and decrease in PNI, lymphocyte count, platelet count and serum albumin were all potential impacts on recurrence.

Cut-off values for the inflammation based index. ROC analysis was used to determine NLR and PNI cutoff point, the cutoff value of NLR was (1.73) with sensitivity (76.2\%), specificity (46.4\%), PPV (51.6\%) NPV (72.2\%) with Accuracy (0.567) and the cutoff point of PNI was
(11.74) with sensitivity (80\%), specificity (90\%), PPV (92.3\%) NPV (75 \%) with Accuracy (0.852) (Figure 1), and these values were used to calculate the NLR-PNI score for each patients. Subsequently, patients were divided into the NLR-PNI 0, NLR-PNI 1, and NLR-PNI2 groups, will be described above.

Regarding to NLR value our patients were divided into patients with NLR $>1.73$ and patients with $\mathrm{NLR}<1.73$ and comparison between these patients at three months post RFA are shown in Table 3. There was statistically significant increase in number of Portahepatis LN, number \&size of tumor in NLR $>1.73$ group than NLR $<1.73$ group with $\mathrm{p}<0.05^{*}$. There was a statistically significant association between child-turcotte-pugh and NLR after RFA as there was increase in number of patients with child A in NLR $<1.73$ group in comparison to NLR $>1.73$ group with $\mathrm{p}<0.05$. Also there was a statistically significant decrease in PNI, lymphocyte number, platelets Number, serum albumin after RFA in NLR >1.73 group in comparison to NLR <1.73 group. While, there were statistically significant increase in PNLR and PLR and change of PNLR in NLR $>1.73$ group when compared to NLR $<1.73$ groupwith $\mathrm{p}<0.001$.

As regard NLR-PNI score our patients were assigned into NLR-PNI 0, NLR-PNI1, and NLR-PNI 2 groups and comparison between these groups are shown in Table 4 where there was a statistically significant increase in number of Portahepatis LN, number \&size of tumor in (NLR-PNI 2) group than (NLR-PNI 0).

Also there was a statistically significant decrease in lymphocyte number, platelets number and serum albumin in (NLR-PNI 2) in comparison to (NLR-PNI 1) and (NLR-PNI 0). Also there was a statistically significant increase in change of PNLR in (NLR-PNI 2) in comparison to (NLR-PNI 1) and (NLR-PNI 0). (Table 5)

Table 1. Demographic and Laboratory data of this study

\begin{tabular}{|l|c|}
\hline Variables & No. /Mean \pm SD \\
\hline Age & $59.52 \pm 7.28$ \\
\hline Female/male & $9 / 41$ \\
\hline Plat Number/ L & $116.80 \pm 57.96$ \\
\hline size (cm)Median(IQR) & $4(2.55-8.6)$ \\
\hline AFP Median (IQR) (ng/ml) & $25(8-95)$ \\
\hline Number of tumour Solitary/ Multiple & $33 / 17$ \\
\hline Baseline NLR & $2.17 \pm 1.18$ \\
\hline Baseline PLR & $0.08 \pm 0.05$ \\
\hline Baseline PNI & $11.38 \pm 3.01$ \\
\hline Baseline PNLR & $268.09 \pm 271.19$ \\
\hline Post RFA NLR & $1.95 \pm 1.00$ \\
\hline Post RFA PLR & $0.07 \pm 0.03$ \\
\hline Post RFA PNI & $11.91 \pm 4.20$ \\
\hline Post RFA PNLR & $214.75 \pm 131.62$ \\
\hline Change in PNLR Decrease/increase & $28 / 22$ \\
\hline
\end{tabular}

Table 2. Baseline inflammatory markers before RFA

\begin{tabular}{|c|c|c|}
\hline \multicolumn{2}{|c|}{} & No. $=50$ \\
\hline NLR & Median(IQR) & $1.97(1.46-2.53)$ \\
\hline PLR & Median(IQR) & $0.07(0.07-0.10)$ \\
\hline PNLR & Median(IQR) & $192.6(191.02-345.16)$ \\
\hline PNI & Median(IQR) & $10.9(10.5-12.2)$ \\
\hline
\end{tabular}

NLR: neutrophil-to-lymphocyte ratio PLR: platelet to lymphocyte ratio, PNI: prognostic nutritional index, PNLR: platelet multiple neutrophil to lymphocyte ratio. 
Table 3. Factors associated with postoperative recurrence as regard Demographic characteristics and Laboratory investigation

\begin{tabular}{|c|c|c|c|c|c|}
\hline & \multicolumn{4}{|c|}{ Recurrence } & \multirow{3}{*}{ Sig. } \\
\hline & \multirow{2}{*}{$\begin{array}{c}\text { No recurrence } \\
\mathbf{N}(20)\end{array}$} & \multirow{2}{*}{$\begin{array}{l}\text { Recurrence } \\
\text { N(30) } \\
\text { Mean } \pm \text { SD }\end{array}$} & \multicolumn{2}{|c|}{ T-test } & \\
\hline & & & $\mathbf{T}$ & P-value & \\
\hline Age & $58.19 \pm 7.92$ & $60.48 \pm 6.76$ & 1.101 & 0.277 & NS \\
\hline $\begin{array}{l}\text { Sex } \\
\text { Female/ Male }\end{array}$ & $3 / 17$ & $6 / 24$ & 0.203 & 0.652 & NS \\
\hline $\begin{array}{l}\text { PortahepatisLN after } 3 M \\
\text { Negative/ Positive }\end{array}$ & $15(75 \%) / 5(25 \%)$ & $14(46.7 \%) / 16(53.3 \%)$ & 3.955 & $0.05^{*}$ & $\mathrm{~S}$ \\
\hline $\begin{array}{l}\text { number of tumar } \\
\text { Soliturty/ Multiple }\end{array}$ & $14(70 \%) / 6(30 \%)$ & $11(36.7 \%) / 19(63.3 \%)$ & 5.333 & $0.05^{*}$ & S \\
\hline Size of tumar & $4.22 \pm 2.5$ & $8.08 \pm 5.7$ & 2.845 & $0.05^{*}$ & $\mathrm{~S}$ \\
\hline \multicolumn{6}{|l|}{ child-turcotte-pugh } \\
\hline A5 & $4 \pm 28.6$ & $14 \pm 38.9$ & 0.466 & 0.495 & NS \\
\hline A6 & $1 \pm 7.1$ & $14 \pm 38.9$ & 4.837 & $<0.05^{*}$ & $\mathrm{~S}$ \\
\hline B7 & $8 \pm 57.1$ & $4 \pm 11.1$ & 11.710 & $<0.001^{* *}$ & HS \\
\hline B8 & $1 \pm 7.1$ & $4 \pm 11.1$ & 0.176 & 0.675 & NS \\
\hline Lymphocyte/ L & $2269.05 \pm 543.79$ & $1384.80 \pm 653.93$ & 5.055 & $<0.001^{* *}$ & HS \\
\hline Plat Number/ L & $147.81 \pm 54.15$ & $88.34 \pm 45.15$ & 4.227 & $<0.001^{* *}$ & HS \\
\hline PLR before ablation & $0.11 \pm 0.06$ & $0.06 \pm 0.03$ & 3.879 & $<0.001^{* *}$ & $\mathrm{HS}$ \\
\hline PLR after ablation & $0.07 \pm 0.02$ & $0.07 \pm 0.04$ & 0.506 & 0.615 & NS \\
\hline PNLR before ablation & $408.38 \pm 359.30$ & $174.57 \pm 130.62$ & 3.268 & $0.05^{*}$ & $\mathrm{~S}$ \\
\hline PNLR after ablation & $220.53 \pm 98.05$ & $210.57 \pm 153.00$ & 0.262 & 0.795 & NS \\
\hline $\begin{array}{l}\text { Change of PNLR } \\
\text { Decrease/increase }\end{array}$ & $17 / 3$ & $11 / 19$ & 11.377 & $<0.001^{* *}$ & HS \\
\hline NLR before ablation & $1.79 \pm 0.83$ & $2.74 \pm 1.41$ & 2.985 & $<0.05^{*}$ & $\mathrm{~S}$ \\
\hline NLR after ablation & $1.85 \pm 0.84$ & $2.7 \pm 1.38$ & 2.985 & $<0.001^{* *}$ & HS \\
\hline Albumin (g/dl) & $3.61 \pm 0.62$ & $2.78 \pm 0.64$ & 4.593 & $0.05^{*}$ & $\mathrm{~S}$ \\
\hline AFP Median (IQR) (ng/ml) & $10(6-35)$ & $14(10-42)$ & 1.735 & 0.102 & NS \\
\hline PNI before ablation & $10.53 \pm 1.76$ & $11.95 \pm 3.53$ & 1.665 & 0.102 & NS \\
\hline PNI after ablation & $14.95 \pm 2.95$ & $9.70 \pm 3.55$ & 5.529 & $<0.001^{* *}$ & HS \\
\hline
\end{tabular}

Table 4. Comparison between patients with increase NLR and patients with decrease NLR At three months post RFA

\begin{tabular}{|c|c|c|c|c|c|}
\hline & \multicolumn{2}{|c|}{ NLR } & \multicolumn{2}{|c|}{ Chi-square } & \multirow{2}{*}{ Sig } \\
\hline & $<1.73$ No (29) & $>1.73$ No (21) & $\mathrm{X} 2$ & P-value & \\
\hline $\begin{array}{l}\text { Portahepatis LN after } \\
\text { Negative/ Positive }\end{array}$ & $22(75.9 \%) / 7(24.1 \%)$ & $7(33.3 \%) / 14(66.7 \%)$ & 9.043 & $<0.05^{*}$ & S \\
\hline $\begin{array}{l}\text { number of tumar } \\
\text { Solitary/ Multiple }\end{array}$ & $19(65.5 \%) / 10(34.5 \%)$ & $6(28.6 \%) / 15(71.4 \%)$ & 6.650 & $<0.05^{*}$ & $\mathrm{~S}$ \\
\hline size cm( Mean \pm SD) & $4.76 \pm 3.32$ & $8.81 \pm 6.13$ & 3.015 & $<0.05^{*}$ & S \\
\hline $\begin{array}{l}\text { HCV } \\
\text { Negative/ Positive }\end{array}$ & 11(37.9\%)/18(62.1\%) & $5(23.8 \%) / 16(76.2 \%)$ & 1.116 & 0.291 & NS \\
\hline \multicolumn{6}{|c|}{ Child-turcotte-pugh after 3M } \\
\hline A5 & $14(48.3 \%)$ & $4(19 \%)$ & 4.516 & $<0.05^{*}$ & $\mathrm{~S}$ \\
\hline A6 & $10(34.5 \%)$ & $5(23.8 \%)$ & 0.661 & 0.416 & NS \\
\hline B7 & $5(17.2 \%)$ & $7(33.3 \%)$ & 1.729 & 0.189 & NS \\
\hline B8 & $0(0.0)$ & $5(23.8 \%)$ & 7.672 & $<0.05^{*}$ & $\mathrm{~S}$ \\
\hline WBCs / L & $5.91 \pm 1.95$ & $5.92 \pm 2.41$ & 0.022 & 0.983 & NS \\
\hline neutrophil / L & $2900.85 \pm 1033.00$ & $3491.55 \pm 1475.52$ & 1.667 & 0.102 & NS \\
\hline Lymphocyte/L & $2138.87 \pm 659.87$ & $1227.72 \pm 505.35$ & 5.297 & $<0.001$ & HS \\
\hline Plat Number/ L & $127.62 \pm 58.16$ & $93.57 \pm 49.98$ & 2.165 & $<0.05$ & $\mathrm{~S}$ \\
\hline PLR & $0.06 \pm 0.02$ & $0.08 \pm 0.04$ & 2.403 & $<0.05$ & S \\
\hline PNLR & $174.7 \pm 100.38$ & $269.96 \pm 151.07$ & 2.678 & $<0.05$ & $\mathrm{~S}$ \\
\hline $\begin{array}{l}\text { Change of PNLR } \\
\text { Decrease/increase }\end{array}$ & $21 / 8$ & $7 / 14$ & 7.550 & $<0.05$ & S \\
\hline Albumin (g/dl) & $3.46 \pm 0.60$ & $2.67 \pm 0.69$ & 4.323 & $<0.001$ & HS \\
\hline AFP (ng/ml) & $19(5-35)$ & $23(10-44.5)$ & 1.846 & 0.095 & NS \\
\hline PNI & $14.16 \pm 3.61$ & $8.81 \pm 2.73$ & 5.704 & $<0.001$ & HS \\
\hline
\end{tabular}


Table 5. Comparison of the clinical characteristics of patients with different NLR-PNI score:

\begin{tabular}{|c|c|c|c|c|c|c|c|c|c|c|c|c|c|}
\hline & \multicolumn{13}{|c|}{ NLR-PNI } \\
\hline & \multicolumn{2}{|c|}{ NLR-PNI 0} & \multicolumn{2}{|c|}{ NLR-PNI 1} & \multicolumn{2}{|c|}{ NLR-PNI 2} & \multicolumn{2}{|c|}{ Total } & \multicolumn{5}{|c|}{ Chi-square } \\
\hline & $\mathbf{N}$ & $\%$ & $\mathbf{N}$ & $\%$ & $\mathbf{N}$ & $\%$ & $\mathbf{N}$ & $\%$ & $\mathrm{X} 2$ & P-value & P1 & P2 & P3 \\
\hline $\begin{array}{l}\text { Portahepatis LN } \\
\text { Negative/ Positive }\end{array}$ & \multicolumn{2}{|c|}{$19 / 5$} & \multicolumn{2}{|c|}{$4 / 3$} & \multicolumn{2}{|c|}{$6 / 13$} & \multicolumn{2}{|c|}{$29 / 21$} & 9.861 & $<0.05^{*}$ & 0.241 & $<0.05^{*}$ & 0.235 \\
\hline $\begin{array}{l}\text { number of tumar } \\
\text { Solitary/ Multiple }\end{array}$ & \multicolumn{2}{|c|}{$16 / 8$} & \multicolumn{2}{|c|}{$4 / 3$} & \multicolumn{2}{|c|}{$5 / 14$} & \multicolumn{2}{|c|}{$25 / 25$} & 7.073 & $<0.05^{*}$ & 0.643 & $<0.05^{*}$ & 0.143 \\
\hline size of tumar & \multicolumn{2}{|c|}{$4.80 \pm 3.12$} & \multicolumn{2}{|c|}{$4.30 \pm 3.80$} & \multicolumn{2}{|c|}{$9.36 \pm 6.20$} & & & 6.069 & $<0.05^{*}$ & 0.966 & $<0.05^{*}$ & $<0.05^{*}$ \\
\hline $\begin{array}{c}\text { HCV } \\
\text { Negative/ Positive }\end{array}$ & \multicolumn{2}{|c|}{$9 / 15$} & \multicolumn{2}{|c|}{$2 / 5$} & & 14 & \multicolumn{2}{|c|}{$16 / 34$} & 0.654 & 0.721 & 0.664 & 0.437 & 0.908 \\
\hline \multicolumn{14}{|c|}{ child-turcotte-pugh } \\
\hline A5 & \multicolumn{2}{|c|}{$14(58.3 \%)$} & \multicolumn{2}{|c|}{$1(14.3 \%)$} & & $.8 \%)$ & \multicolumn{2}{|c|}{$18(36 \%)$} & \multirow{4}{*}{16.224} & \multirow{4}{*}{$<0.05^{*}$} & \multirow{4}{*}{$<0.05^{*}$} & \multirow{4}{*}{$<0.05^{*}$} & \multirow{4}{*}{0.980} \\
\hline A6 & & & $2(2$ & & & $3 \%)$ & & $30 \%)$ & & & & & \\
\hline B7 & \multicolumn{2}{|c|}{$2(8.3 \%)$} & \multicolumn{2}{|c|}{$3(42.9 \%)$} & & 6.8) & \multicolumn{2}{|c|}{$12(24 \%)$} & & & & & \\
\hline B8 & \multicolumn{2}{|c|}{$0(0.0)$} & \multicolumn{2}{|c|}{$1(14.3 \%)$} & & $.1 \%)$ & \multicolumn{2}{|c|}{$5(10 \%)$} & & & & & \\
\hline
\end{tabular}

Table 6. Comparison of the laboratory investigation of patients with different NLR-PNI score

\begin{tabular}{|c|c|c|c|c|c|c|c|c|c|c|c|}
\hline & \multicolumn{2}{|c|}{ NLR-PNI 0} & \multicolumn{2}{|c|}{ NLR-PNI 1} & \multicolumn{2}{|c|}{ NLR-PNI 2} & \multicolumn{2}{|c|}{ ANOVA } & \multicolumn{3}{|c|}{ Tukey's- test } \\
\hline & Mean & SD & Mean & SD & Mean & SD & $\mathbf{F}$ & $\begin{array}{l}\text { P-value } \\
\end{array}$ & P1 & P2 & P3 \\
\hline WBCs/L & 6.45 & 1.64 & 4.11 & 2.35 & 5.91 & 2.34 & 3.575 & $0.036^{*}$ & $0.05^{*}$ & 0.669 & 0.123 \\
\hline neutrophil/ L & 3183.17 & 838.39 & 3044.66 & 2646.48 & 3144.13 & 1037.55 & 0.032 & 0.969 & 0.966 & 0.995 & 0.983 \\
\hline Lymphocyte/ L & 2334.71 & 500.52 & 1523.44 & 700.42 & 1111.16 & 364.30 & 34.562 & $<0.001^{* *}$ & $<0.001$ & $<0.001$ & 0.145 \\
\hline Plat Number/ L & 141.88 & 53.26 & 61.71 & 15.25 & 96.26 & 51.86 & 8.935 & $<0.001^{* *}$ & $<0.001$ & $<0.05^{*}$ & 0.264 \\
\hline Albumin (g/dl) & 3.63 & 0.46 & 2.76 & 0.86 & 2.64 & 0.61 & 16.825 & $<0.001^{* *}$ & $<0.05^{*}$ & $<0.001$ & 0.888 \\
\hline AFP (ng/ml) & 168.59 & 320.95 & 14.97 & 8.37 & 419.23 & 1821.39 & 0.356 & 0.702 & 0.747 & 0.803 & 0.961 \\
\hline PLR befor ablation. & 0.10 & 0.06 & 0.06 & 0.02 & 0.07 & 0.04 & 2.275 & 0.114 & 0.219 & 0.192 & 0.909 \\
\hline PLR after a Blation & 0.06 & 0.02 & 0.05 & 0.02 & 0.09 & 0.04 & 6.297 & $<0.05^{*}$ & 0.528 & $<0.05^{*}$ & 0.011 \\
\hline PNLR befor ablation & 350.20 & 346.24 & 118.24 & 80.04 & 219.59 & 157.10 & 2.638 & 0.082 & 0.110 & 0.247 & 0.660 \\
\hline PNLR after ablation & 195.63 & 97.96 & 109.86 & 62.48 & 277.55 & 157.06 & 5.488 & $<0.05^{*}$ & 0.235 & 0.081 & 0.008 \\
\hline $\begin{array}{l}\text { Change of PNLR } \\
\text { Decrease/ Increase }\end{array}$ & \multicolumn{2}{|c|}{$19 / 5$} & \multicolumn{2}{|c|}{$3 / 4$} & \multicolumn{2}{|c|}{$6 / 13$} & 10.317 & $<0.05^{*}$ & 0.063 & $0.05^{*}$ & 0.592 \\
\hline
\end{tabular}

NLR-PNI $0=($ NLR $<1.73+$ PNI $>11.74)$, NLR-PNI $1=$ (NLR $>1.73$ or PNI $<11.74)$, NLR-PNI $2=$ (NLR $>1.73+$ PNI $<11.74)$. When each group compared with each other. P1= NLR-PNI 0\& NLR-PNI 1, P2= NLR-PNI 0\& NLR-PNI 2, P3= NLR-PNI 1\& NLR-PNI 2.

Table 7. Factors associated with post RFA survival in the multivariate analysis

\begin{tabular}{|l|c|c|c|c|c|c|}
\hline \multirow{2}{*}{ Regression } & \multirow{2}{*}{ HR } & \multicolumn{2}{|c|}{$\mathbf{9 5 \%}$ Confidence Interval } & \multirow{2}{*}{ T } & \multirow{2}{*}{ P value } & \multirow{2}{*}{ Sig } \\
\cline { 3 - 4 } & & Lower & Upper & & \\
\hline Plat Number After ablation & 110.78 & 96.93 & 129.97 & 0.303 & 0.764 & NS \\
\hline PLR After ablation & 0.07 & 0.06 & 0.08 & 2.066 & $<0.05^{*}$ & S \\
\hline NLR & 2.09 & 1.88 & 2.55 & 2.403 & $<0.05^{*}$ & S \\
\hline Albumin(g/dl) After ablation & 3.13 & 2.91 & 3.34 & 2.589 & $<0.05^{*}$ & S \\
\hline AFP (ng/ml) After ablation & 59.20 & -99.74 & 638.78 & 2.448 & $<0.05^{*}$ & S \\
\hline
\end{tabular}
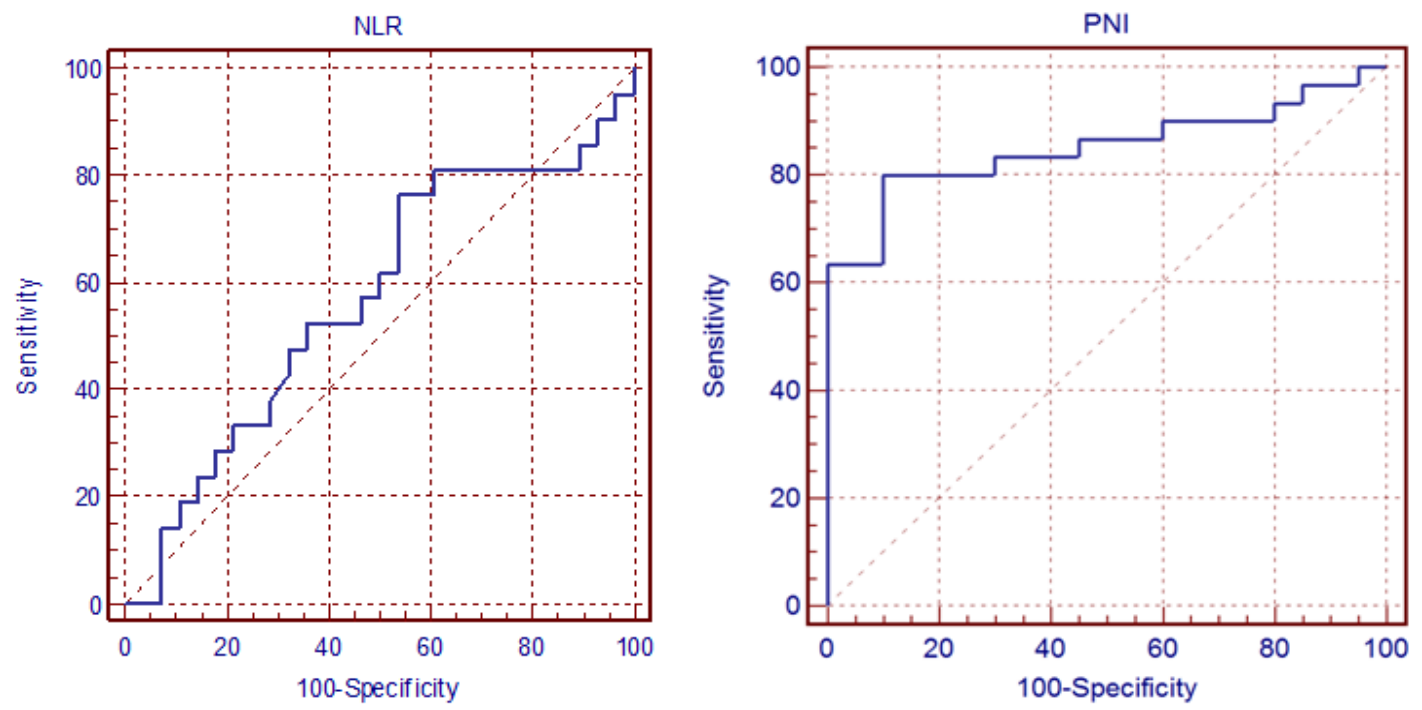

Figure 1. ROC curve of NLR Showed ROC curve of PNI 
In the current study analysis was performed according to the multivariate Cox proportional hazards model to identify risk factors associated with post RFA survival by univariant and multivariant analysis. In univariant analysis high post RFANLR and PLR were associated with mortality after RFA with HCC. Also, high AFP level (HR $=59.2,95 \%$; CI $=$-99.74-638.78; $\mathrm{P}=0.021)$, Albumin $(\mathrm{HR}$ 3.13, 95\%; CI = 2.91 - 3.34: $\mathrm{P}=0.010)$, NLR $(\mathrm{HR}=2.09$, 95\%; $\mathrm{CI}=1.88-2.55: \mathrm{P}=0.018)$ and PLR $(\mathrm{HR}=0.07 ; 95 \%$; $\mathrm{CI}=0.06-0.08 ; \mathrm{P}=0.048)$. Were demonstrated as independent prognostic factor for poor survival(Table 6).

\section{Discussion}

There is a strong association between inflammation and cancer. Systemic Inflammatory Response (SIR) was considered as an important factor influences HCC recurrence and/or overall survival for patients with HCC following liver resection, liver transplantation, radio frequency ablation [8].

Several indicators of systemic inflammation such as C-reactive protein, neutrophil-to-lymphocyte ratio (NLR), lymphocyte-to-monocyte ratio, platelet-to-lymphocyte ratio (PLR) and platelet neutrophil-to-lymphocyte ratio (PNLR) have been used for evaluation of inflammatory status [16]. However, the prognostic power of these inflammation-based prognostic systems is still under debate. Platelet, neutrophil, and lymphocyte have their own unique role in influencing recurrence for patients with HCC [17].

This study was conducted to evaluate the prognostic performance of NLR in Egyptian patients has HCC and was treated with RFA.

In this study Radiological characteristics was assessed before and after RFA and showed that a tumor size more than $8 \mathrm{~cm}$, multiple tumor $(19,63.3 \%)$ and incidence of Portahepatis LN (16, 53.3\%), significant increase in patients with recurrence HCC, and also found child-turcotte-pugh was prognostic parameter for recurrence HCC after RFA and there was increase in number of patients with child score "B" in recurrence group in comparison to non-recurrence group. Chen et al. (2012) [18] reported that The CTP class had a definite effect upon disease outcome. Also they found that tumour size, tumor multiplicity and AFP had independent determining effects on OS and/or tumor recurrence, which is consistent with previous reports indicating that these tumor characteristics are associated with vascular invasion and a more aggressive phenotype. This finding reinforced the importance of rigid surveillance protocols for high-risk patients to detect HCC at an early stage.

One of the major contributing factors for HCC development, recurrence and metastasis is the imbalance of inflammatory response [19]. An elevated ratio of peripheral neutrophils-to-lymphocytes (NLR) has been recognized as a prognostic indicator in various cancers. However, the consistency and magnitude of the prognostic impact of NLR are unclear. NLR might be a readily available and inexpensive objective prognostic index that could be used in daily oncologic clinical practice and could help to stratify patients in clinical trial [10].
Because blood neutrophil and lymphocyte counts are routinely checked during clinical practice, our study evaluate the baseline inflammatory markers before RFA and found the median of NLR, PLR and PNLR were 1.97 (0.41 - 7.1), 0.07 (0.02 - 0.3), 192.6(44.39 - 1622.4) respectively. Also in our study the baseline PNI was median10.9 (10.5- 20.5). In Recurrence group of patients there was a statistically significant decrease in lymphocyte number, platelet number, serum albumin and PNI in comparison to non-recurrence group, but there was a statistically significant increase in NLRin patients with recurrence HCC.

Chien et al., (2017) [20] assess the role of NLR on recurrent HCC, the association between recurrence-to-death, survival and the change of NLR from preoperative to recurrent status and they found unfavorable outcome noticed for recurrent HCC patients with a new or persistent elevation of NLR.

Also they reported that High NLR at recurrence predicted inferior recurrence-to-death survival. In patients with initial low NLR, a higher level of NLR at recurrence discriminated patients with poor recurrence-to-death survival, which was similar to the patients with initial high NLR. The levels of NLR at tumor recurrence deeply influenced post-recurrent survival no matter whether the initial NLR was low or high.

In our study there was statistically significant increase in number of patients with increase of PNLR in Recurrence HCC. Jin et al. (2017) [21] concluded that suggested change of postoperative PNLR, a simple and easily calculated inflammatory marker, can independently predict postoperative OS and recurrence free survival (RFS) for patients with BCLC A stage HCC after liver resection. Change of postoperative PNLR helps to identify patients at high risk of postoperative recurrence.

In our study, the NLR and PNI cut-off values were determined using receiver operating characteristic (ROC) curves according patient recurrence the cut value of NLR is 1.73 and PNI is 11.74 .

Of the 50 patients in our study 29 has an NLR $<1.73$ and 21 patients has an NLR $\geq 1.73$. Most of the characteristics of two groups were similar. Patients in high NLR group had statistically significant higher in number of Portahepatis LN ( $\mathrm{P}<0.05)$, maximum diameter of tumor ( $\mathrm{P}<0.05)$ multiple nodules $(\mathrm{P}<0.001)$, and worse childturcotte-pugh $(\mathrm{P}<0.05)$.

Recently Chien et al. (2017) [20] reported that according to NLR cut-off value, the patients with NLR $>2.5$ had larger tumor size, higher histology grade, and higher rates of tumor multiplicity and vascular invasion. Also Tsunematsu et al. (2017) [22] who did meta-analysis revealed that high NLR is associated with high-grade HCC, including vessel invasion, multiple tumors and high AFP levels, which are all associated with tumor recurrence and poor prognosis. Li et al. (2017) [23] also, found that the most of patients has NLR $\geq 2.9$ had significantly higher serum AFP level $(\mathrm{P}=0.038)$, maximum diameter tumor, worse Child-Pugh score, longer operative time $(\mathrm{P}=0.011)$, and shorter surgical margin.

In our study patients has NLR $\geq 1.73$ were associated with statistically significant decrease of lymphocyte 
number, platelet number, serum albumin and PNI with $(p=<0.001)$, but there was a statistically significant increase in PNLR, PLR and change in PNLR. Jin et al. (2017) [21] confirmed the prognostic power of change of PNLR was better than NLR, PLR, and PNI.

Some inflammation-based prognostic systems, such as NLR and PLR, were confirmed to influence the prognosis of HCC after RFA by previous investigations [24].

The presence of an inflammatory response is considered pathogenic in the development of cancer-associated malnutrition, and leads to a poor performance status and increased mortality in cancer patients [25].

As the prognostic values of the NLR and PNI are stable in patients with early-stage HCC, we evaluated whether a combination of these scores could optimize the selection of patients who would benefit from RFA among a patient subgroup with varying tumors burdens and variable predicted survival outcomes.

The NLR-PNI score was then generated by combining the NLR score with the PNI score. The NLR and PNI cutoff values were determined using receiver operating characteristic (ROC) curves.

The NLR-PNI score was calculated and revealed that 24 patients (48\%), 7(14\%), and19 (38\%) patients were assigned into NLR-PNI 0, NLR-PNI 1 and NLR-PNI 2 respectively.

Patients in (NLR-PNI 2) group had relatively largest tumor size, more multiplicity of tumor and increase incidence of Portahepatis LN compared with other groups $(\mathrm{P}<0.05)$. Also the three groups had significantly different in serum albumin and Patients in (NLR-PNI 0) had the highest level $(\mathrm{P}<0.001)$.

Also our results found that patients in the (NLR-PNI 2) group had statistically decreased in lymphocyte number, platelet numbers and statistically significant increase in PLR and change of PNLR.

Our results indicated that the combined NLR-PNI score could better reflect the systemic inflammatory response for patients with HCC after RFA, compared with either score alone.

Liu et al. (2017) [26] who was the first one evaluated the ability of the combined NLR-PNI score to predict the outcomes of patients with early stage HCC after RFA and reported that after dividing their patients into three groups according to NLR-PNI scores, those patients with high combined scores had progressively worse outcomes relative to those with lower scores, and these differences were largely significant.

Previous studies hypothesized that the PNI reflects the patient's general status, including their immune-nutritional status, liver function, and immune activity, rather than the malignant potential of the tumor [27].

In our study analysis was performed to identify risk factor associated with post RFA survival by univariant and multivariant analysis. In univariant analysis high post RFA NLR and PLR were associated with mortality after RFA with HCC. Also, high AFP level, serum albumin, NLR and PLR were demonstrated as independent prognostic factor for poor survival. Also, our results demonstrated that there was no statistically significant association between survival duration and reported levels of NLR.
Jin et al. (2017) [21] reported by uninvariant analysis, multiple tumor, micro vascular invasion (MVI), high preoperative AFP level, differentiation, intra operative transfusion, tumour size, post-operative NLR and change of PNLR were associated with mortality after liver resection for patients with HCC within the BCLC stage A. Also, reported by multivariant analysis that only MVI, post-operative NLR and change of PNLR are independent prognostic factor for poor OS.

In contrast to our results, Bertuzzo et al. (2017), (28), reported that NLR did not affect overall survival in patients with HCC who underwent OLT.

\section{Conclusion}

High blood NLR after RFA is a predictor for worse survival and also can predict recurrence of HCC. Higher NLR-PNI score predict a worse prognosis in patients who underwent RFA.

\section{References}

[1] White DL, Thrift AP, Kanwal F, Davila J, El-Serag HB. Incidence of hepatocellular carcinoma in all 50 United States, from 2000 through 2012. Gastroenterology 2017; 152: 812-820, e5.

[2] Hao-Chien Hung, Jin-Chiao Lee, Chih-Hsien Cheng, Tsung-Han Wu, Yu-Chao Wang, Chen-Fang Lee, et al. (2017). Impact of neutrophil to lymphocyte ratio on survival for hepatocellular carcinoma after curative resection, J Hepatobiliary Pancreat Sci 24:559-569.

[3] She WH, Cheung TT, Jenkins CR, et al. (2016). Clinical applications of high-intensity focused ultrasound. Hong Kong Medical Journal. Aug; 22(4):382-92.

[4] Lü MD, Kuang M, Liang LJ, Xie XY, Peng BG, Liu GJ, Li DM, Lai JM and Li SQ. Surgical resection versus percutaneous thermal ablation for early-stage hepatocellular carcinoma: A randomized clinical trial. Zhonghua Yi Xue Za Zhi 86: 801-805, 2006 (In Chinese).

[5] Chen MS, Li JQ, Zheng Y, Guo RP, Liang HH, Zhang YQ, Lin XJ and Lau WY. A prospective randomized trial comparing percutaneous local ablative therapy and partial hepatectomy for small hepatocellular carcinoma. Ann Surg 243: 321-328, 2006.

[6] Livraghi T, Meloni F, Di Stasi M, Rolle E, Solbiati L, Tinelli C and Rossi S. Sustained complete response and complications rates after radiofrequency ablation of very early hepatocellular carcinoma in cirrhosis: Is resection still the treatment of choice? Hepatology 47: 82-89, 2008.

[7] Kao WY, Chiou YY, Hung HH, Chou YH, Su CW, Wu JC, Huo TI, Huang YH, Lin HC and Lee SD. Risk factors for long-term prognosis in hepatocellular carcinoma after radiofrequency ablation therapy: The clinical implication of aspartate aminotransferase-platelet ratio index. Eur J Gastroenterol Hepatol 23: 528-536, 2011.

[8] Dan J, Zhang Y, Peng Z, et al. (2013). Postoperative neutrophil-to lymphocyte ratio change predicts survival of patients with small hepatocellular carcinoma undergoing radiofrequency ablation. PLoS One. 8: e58184.

[9] Chan AW, Chan SL,Wong GL, et al. (2015). Prognostic Nutritional Index (PNI) predicts tumor recurrence of very early/early stage hepatocellular carcinoma after surgical resection. Ann Surg Oncol. 22: 41: 38-48.

[10] Motomura T, Shirabe K, Mano Y, et al. (2013). Neutrophillymphocyte ratio reflects hepatocellular carcinoma recurrence after liver transplantation via inflammatory microenvironment. J Hepatol. 58(1): 58-64.

[11] Bruix J and Sherman M (2011): Management of Hepatocellular Carcinoma: An update. American Association for the Study of Liver Diseases (AASLD) practice guideline. Hepatol; 53(3): 1020-1022. 
[12] Cholongitas E, Papatheodoridis GV, Vangeli M, et al. (2005). "Systematic review: The model for end-stage liver disease--should it replace Child-Pugh's classification for assessing prognosis in cirrhosis?". Aliment Pharmacol Ther; 22: 1079-1089.

[13] Liovet JM, Bustamante J, Castells A, et al. (1999). Natural history of untreated nonsurgical hepatocellular carcinoma: rationale for the design and evaluation of therapeutic trials. Hepatology; 29: 62-67.

[14] Xiao, G. Q., Liu, C., Liu, D. L., Yang, J. Y. \& Yan, L. N. Neutrophil-lymphocyte ratio predicts the prognosis of patients with hepatocellular carcinoma after liver transplantation. World J Gastroenterol. 19, 8398-8407 (2013).

[15] Onodera, T., Goseki, N. \& Kosaki, G. [Prognostic nutritional index in gastrointestinal surgery of malnourished cancer patients]. Nihon Geka Gakkai Zasshi. 85, 1001-1005 (1984).

[16] Le PH, Liang KH, Chang ML, et al. (2017). Clinical Predictors for Neutrophil-to-Lymphocyte Ratio Changes in Patients with Chronic Hepatitis B Receiving Peginterferon Treatment. in vivo; 31(4): 723-9.

[17] Xiao WK, Chen D, Li SQ, et al. (2014). Prognostic significance of neutrophillymphocyte ratio in hepatocellular carcinoma: a metaanalysis. BMC Cancer. 14:117.

[18] Chen TM, Lin CC, Huang PT, Wen CF (2012). Neutrophil-tolymphocyte ratio associated with mortality in early hepatocellular carcinoma patients after radiofrequency ablation. J Gastroenterol Hepatol. 27:553-61.

[19] Budhu A, Forgues M, Ye QH, et al. (2006). Prediction of venous metastases, recurrence, and prognosis in hepatocellular carcinoma based on a unique immune response signature of the liver microenvironment. Cancer Cell 10(2): 99-111.

[20] Chien HC, Lee JC, Cheng CH, et al. (2017). Impact of neutrophil to lymphocyte ratio on survival for hepatocellular carcinoma after curative resection. Journal of Hepato-biliary-pancreatic Sciences; 24(10): 559-69.

[21] Jin C, Li C, Peng W, et al. (2017). Changes of platelet times neutrophil to lymphocyte ratio predict BCLC stage A hepatocellular carcinoma survival. Medicine; 96(33).

[22] Tsunematsu S, Suda G, Yamasaki K, et al. (2017). Combination of neutrophil-to-lymphocyte ratio and early des- $\gamma$ carboxyprothrombin change ratio as a useful predictor of treatment response for hepatic arterial infusion chemotherapy against advanced hepatocellular carcinoma. Hepatology Research; 47(6):533-41.

[23] Li J, Liao Y, Suo L, et al. (2017). A novel prognostic indexneutrophil times $\gamma$-glutamyl transpeptidase to lymphocyte ratio (N $\gamma \mathrm{LR})$ predicts outcome for patients with hepatocellular carcinoma. Scientific Reports; 7(1): 9229.

[24] Chen Q, Dai Z, Yin D, et al. (2015). Negative impact of preoperative platelet lymphocyte ratio on outcome after hepatic resection for intrahepatic cholangiocarcinoma. Medicine (Baltimore); 94: e574.

[25] Pinato DJ, North BV, Sharma R (2012). A novel, externally validated inflammation-based prognostic algorithm in hepatocellular carcinoma: the prognostic nutritional index (PNI). British journal of cancer. 106(8):1439.

[26] Liu C, Li L, Lu WS, et al. (2017). Neutrophil-lymphocyte ratio plus prognostic nutritional index predicts the outcomes of patients with unresectable hepatocellular carcinoma after transarterial chemoembolization. Scientific Reports; Oct 24; 7(1): 13873.

[27] Okamura Y, Sugiura T, Ito T, et al. (2017): The optimal cut-off value of the preoperative prognostic nutritional index for the survival differs according to the TNM stage in hepatocellular carcinoma. Surgery Today, 47(8): 986-993.

[28] Bertuzzo VR, Cescon M, Odaldi F, et al. (2017). Actual risk of using very aged donors for unselected liver transplant candidates. Annals of Surgery; 265(2):388-96.

C The Author(s) 2019. This article is an open access article distributed under the terms and conditions of the Creative Commons Attribution (CC BY) license (http://creativecommons.org/licenses/by/4.0/). 